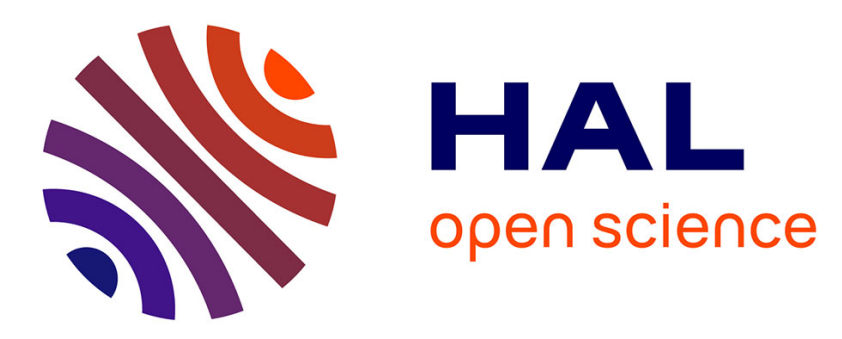

\title{
A typology of distance-based measures of spatial concentration
}

\author{
Eric Marcon, Florence Puech
}

\section{To cite this version:}

Eric Marcon, Florence Puech. A typology of distance-based measures of spatial concentration. Regional Science and Urban Economics, 2016, 62, pp.56-67. 10.1016/j.regsciurbeco.2016.10.004 . halshs-00679993v6

\section{HAL Id: halshs-00679993 \\ https://shs.hal.science/halshs-00679993v6}

Submitted on 18 Oct 2016

HAL is a multi-disciplinary open access archive for the deposit and dissemination of scientific research documents, whether they are published or not. The documents may come from teaching and research institutions in France or abroad, or from public or private research centers.
L'archive ouverte pluridisciplinaire HAL, est destinée au dépôt et à la diffusion de documents scientifiques de niveau recherche, publiés ou non, émanant des établissements d'enseignement et de recherche français ou étrangers, des laboratoires publics ou privés. 


\title{
A Typology of Distance-Based Measures of Spatial Concentration
}

\author{
Eric Marcon ${ }^{1}$, Florence Puech ${ }^{2 *}$
}

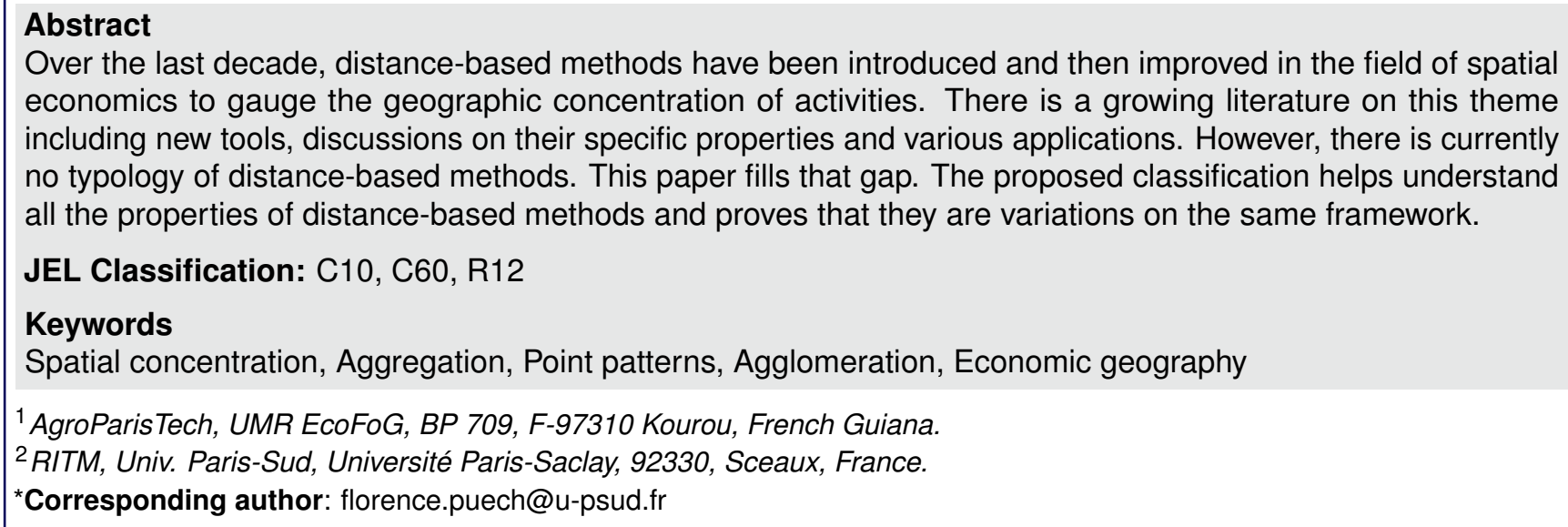




\section{Introduction}

In the article on "spatial economics" in the New Palgrave Dictionary of Economics, Gilles Duranton wrote "On the empirical front, a first key challenge is to develop new tools for spatial analysis. With very detailed data becoming available, new tools are needed. Ideally, all the data work should be done in continuous space to avoid border biases and arbitrary spatial units." (Duranton, 2008). In recent years, economists have made every effort in that direction. Measurement of the spatial concentration of activities is certainly one of the most striking examples and has been considerably renewed in the last decade with the development of distance-based methods (Combes et al., 2008). To present the motivation for the use of distance-based methods briefly, let us say that economists traditionally employ disproportionality methods (terminology used by Bickenbach and Bode, 2008) defined on a discrete definition of space. In the latter, the territory being analyzed is divided in several exclusive zones (e.g. a country is divided in turn into regions) and the spatial concentration of activities is evaluated at a given level of observation with the Gini (1912), the Ellison and Glaeser (1997) or the entropy indices of overall localization (Cutrini, 2009), for example. However, the issues arising from discrete spaces are now well known and linked to the Modifiable Areal Unit Problem - MAUP (Arbia, 1989; Openshaw and Taylor, 1979): the position of the zoning boundaries and level of observation have an impact (Briant et al., 2010). A first tentative to limit the MAUP's effects is to combine discrete-based measures with autocorrelation measures. The motivation is the following: results of spatial concentration provided by discrete-based measures are not affected by the permutation of zones (see Arbia, 2001b, for an illustrative example). As autocorrelation measures evaluate the degree of similarity between zones, they can bring complementary results to the spatial concentration estimates (Guillain and Le Gallo, 2010). Some authors also try to correct in some extent aspatial concentration results by integrating the degree of autocorrelation to the spatial concentration indices (Guimarães et al., 2011). This approach can be of interest if data is only available at the aggregated level of the zone. A second way of research has been undoubtedly more explored since a decade. This second approach does not limit the effects of the MAUP but solves the MAUP. The basic idea is to remove any zoning of space. The development of spatial concentration indices is compulsory to take more effective account of geography (Marcon and Puech, 2003). This encourages the development of distance-based methods which are continuous functions of space. Distance-based measures provide information about concentration at all scales simultaneously and do not rely on zoning. In that case, individual data (and not aggregated data) is used. The seminal work by Ripley $(1976,1977)$ introduced the best known of the existing distance-based methods: the $K$ function. The latter was taken up quickly by field scientists in ecology (see handbooks by Diggle, 1983; Cressie, 1993, for instance) but its use remained incidental in economics (Arbia, 1989; Arbia and Espa, 1996; Barff, 1987; Feser and Sweeney, 2000; Sweeney and Feser, 1998) until the works of Marcon and Puech (2003, 2010) and Duranton and Overman $(2002)^{1}$ who introduced an alternative approach.

In this paper, we propose a typology of distance-based methods. There are two main reasons behind our work. First, a great variety of distance-based methods are used by economists today. The varied toolbox provided by these measures may bring some confusion for economists interested in testing a hypothesis rather than a methodology, so a state of the art may be helpful. Second, in this article we provide a unified theoretical framework by showing that all distance-based methods rely on counting the number of neighbors of points, normalizing this number by space or another number of neighbors, averaging the results in the appropriate way and finally normalizing the result. Monte-Carlo simulations of the null hypothesis allow the data to be tested against it and can also solve remaining issues. As a result, if objects (for example plants) attract each other, more neighbors (other plants) will be found around them on average than if they were distributed randomly and independently. In conclusion, these methods are variations on the same framework to gauge spatial concentration. This being the case, this typology can be useful for readers to choose the appropriate distance-based tool to answer their question.

The paper is organized as follows. In the first part, we give a quick presentation of the common framework and basic vocabulary. Then, all the available distance-based measures are introduced. The third part builds a typology of these methods, showing that they follow the same pattern but vary because they assume different theoretical choices. The last part is a discussion of each tool's properties and their relevance to address economic questions.

\footnotetext{
${ }^{1}$ Published as Duranton and Overman (2005).
} 


\section{Basic principles}

Before presenting distance-based measures in detail, we shall propose a general overview of the framework of these functions.

When studying the location of activities, economists document the spatial distribution of one kind of entity $\left(\right.$ points $\left.^{2}\right)$, for example shops with a given activity. Their aim is to detect phenomena of attraction (also called aggregation, agglomeration, localization), repulsion (dispersion) or independence between those entities in a territory. Industries that are spatially concentrated or dispersed are sometimes referred as diverging industries (Barlet et al., 2013). Another field of research analyzes the relations between entities belonging not to one, but to two different groups (co-localization phenomenon). All the tools considered in this review identify the spatial structure of the point distribution. Their particularity resides in their analysis of space: they treat space as continuous and not as a collection of predefined zones. They are based on the distance separating pairs of entities, which is why they are called distance-based methods or distance-based measures. Results are presented as a plot of a function of distance, whose values are meaningful or not, and always compared to an envelope representing the confidence interval of a null hypothesis to be tested. Intratype (or univariate) functions consider a single type of points to address their localization. Intertype (or bivariate) functions are used to characterize co-localization. They are built in the same way. The curve shows the potential attraction or repulsion of two types of points (between two types of shops for example). Moreover, most functions allow weighting points. For example, if one studies the spatial distribution of shops, (s)he can consider shops regardless of its size or (s)he can keep the number of employees of shops in her/his analysis. Loosely speaking, distance-based measures compare the number of neighbors of points of interest to a benchmark corresponding to the null hypothesis of no spatial structure. This can be done in many ways leading to critical choices related to the question to tackle. After Brülhart and Traeger (2005) and Marcon and Puech (2010), we define a typology of the methods:

- The null hypothesis of no spatial structure may be that the points of interest are distributed like the others: more points will be found in dense areas. The alternative null hypothesis is that their density is a function of space, the same everywhere if space is homogeneous or variable if space is inhomogeneous. The null hypothesis generates a benchmark included in the measure. The choice of the benchmark defines relative vs topographic functions. Some functions have no benchmark, so they are called absolute functions: their value is of little interest but they can be compared to the confidence interval of their null hypothesis to detect significant, but not directly quantifiable, patterns.

- The number of neighbors may be considered at a given distance, or up to it. This choice defines density vs cumulative functions.

To give a brief survey, let us consider the map given of figure 1a. Two types of points are distributed on the squared territory: the "cases" and the "controls". The cases will be the points of interest. Figure 1b presents the results of a distance-based measure, the $m$ function, applied to the hypothetical sample of points of figure 1a. The $m$ function (y-axis) is plotted against distance (x-axis). The value $m(r)$ at distance $r$ is the ratio of the number of case-neighbors $r$ apart from each case to the number of all neighbors. A confidence interval is calculated for the following null hypothesis: the cases have the same spatial distribution as the whole point pattern. The center of the confidence interval (denoted CI center on figure $1 \mathrm{~b}$ ) is the expected value of the function according to the null hypothesis. Values of the $m$ function above the confidence interval for a range of distances indicate spatial concentration of cases relatively to controls at these distances. Values of the $m$ function under the confidence interval detect repulsion of cases at these distances. The $m$ function is estimated for all distances: a complete description of interactions between points can be identified and comparisons of the level of spatial concentration is possible. For example, is the spatial degree of concentration of cases in the clusters more important than the one between clusters? The answer is provided by comparing the $m$ values reached at the first and second concentration peaks on figure $1 \mathrm{~b}$. We conclude immediately that the level of the relative spatial concentration of cases inside clusters is the highest.

\footnotetext{
${ }^{2}$ In the entire article, the term "points" refers to the studied entities (shops, plants etc.) of the sample.
} 
Finally note that the $m$ function plotted on figure $1 \mathrm{~b}$ is a relative, density function. It is univariate and, for the sake of simplicity in that example, it has been applied to unweighted points. This short, informal presentation will be detailed in the remainder of the paper.

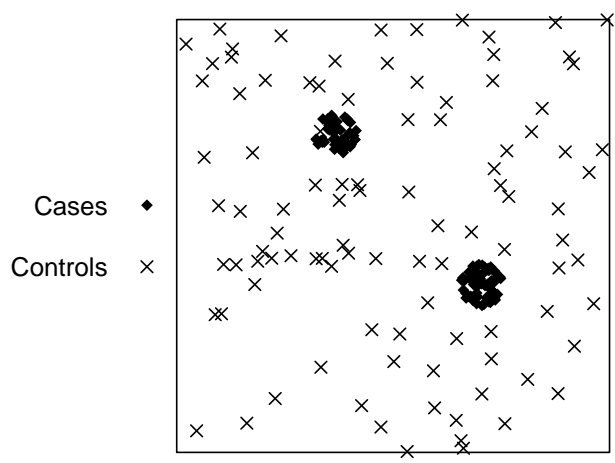

(a) Hypothetical sample with an aggregate distribution of cases and a complete spatial randomness for controls.

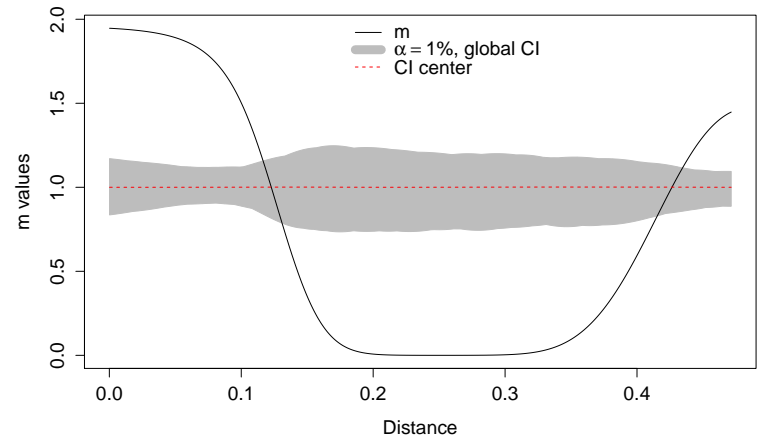

(b) $m$ function results

Figure 1. $m$ function applied to a hypothetical distribution of points (cases are the points of interest)

To go farther in more technical terms, let us say that distance-based methods investigate the spatial structure of point patterns. Their mathematical framework is that of point processes. It is clearly outside the scope of this paper to explore the point process theory (see the first chapter of Møller and Waagepetersen, 2004, for a rigorous introduction), but basic knowledge is required for a good understanding of what follows. Point processes are similar to random variables (the best known are described by their distribution, with parameters) however their output is not a number but a point set in space (often called a point pattern). Practical interest is limited to two-dimensional finite spaces, that is to say points on a map. The observed point set is a realization of an underlying point process for which the law is unknown, so its characterization must be non-parametric: some statistics will be used for tests and even to quantify some properties, but identifying the point process will generally remain impossible. These statistics will be defined according to relevant properties of the point process. Their value will be estimated from the data.

The first property of interest is intensity, the number of points per unit of space observed in a small area. Intensity is denoted $\lambda(x)$, where $x$ is a point of the sample. If intensity is the same throughout the space, it is denoted $\lambda$ and the point process is said to be stationary. The probability of finding a point around $x$ is $\lambda(x) \mathrm{d} x$. Intensity can be estimated by density $\widehat{\lambda}(x)$, the number of observed points per unit of space. In simple cases, points are just counted. If density changes over the space, kernel methods (Diggle, 1985) must be used. They rely on counting points around $x$, up to a chosen distance called bandwidth, while giving a decreasing weight to the further points. Several types of smoothing exist (Silverman, 1986) but Gaussian kernel smoothing is generally employed in spatial economics (see Duranton and Overman, 2005, among others).

\section{Distance-based methods: a brief presentation}

\subsection{The $g$ function}

The second-order property of a point pattern characterizes the relation between points: attraction, repulsion or independence. It is defined as the ratio between the joint probability of finding two points in two places $x$ and $y$, denoted $\lambda(x, y) \mathrm{d} x \mathrm{~d} y$ and the product of the probabilities of finding each of them. For practical purposes, this property is assumed to depend only on the distance between the points (as it does not change with direction, the point process is said to be isotropic). A stationary and isotropic process is called homogeneous. The second-order property is denoted $g(r)=\lambda(x, y) /[\lambda(x) \lambda(y)]$ where $r$ is the distance between two points $x$ and $y$. If points are 
distributed independently, $g(r)=1$. This value corresponds to the benchmark where no interaction between points is detected. If $g(r)>1$, the probability of finding two points $r$ apart is greater than if they were independent and inversely for $g(r)<1$. The first case corresponds to attraction, the second to dispersion.

The following idea, "writing the conditional probability of finding a point around $y$ when a point is actually at $x$ ", is at the origin of all the measures presented here. It results in the following method:

- Some points are chosen as reference points, for example establishments of one given manufacturing industry.

- Neighbors of reference points are counted at distance $r$. Neighbors may be of a different type to the reference points or the same. In the former case, intertype (or bivariate) measures are defined, and intratype (univariate) in the latter.

- The ratio of the average density of neighbors at distance $r$ from reference points to the density of this type of points anywhere is the estimator of $g$, noted $\hat{g}(r)$. It is a location quotient (Florence, 1972).

Estimating $g(r)$ from the data requires a technique to count neighbors at a given distance: kernel functions are used again, but in one dimension this time. A kernel function gives a weight to neighbors at distances around $r$. The closer the distance to $r$, the greater the weight, and the kernel function sums to 1 for all distances. Duranton and Overman (2005, Eq.1) used a Gaussian kernel following Silverman (1986). Its estimator is $k\left(\left\|x_{i}-x_{j}\right\|, r\right)$ where $x_{i}$ and $x_{j}$ are the exact locations of points $i$ and $j .^{3}$

The maximum value of $k\left(\left\|x_{i}-x_{j}\right\|, r\right)$ is reached at the exact distance $r$ between points $i$ and $j$ and decreases according to a Gaussian distribution of standard deviation $h$ if the distance between points deviates from $r$. We can define:

$$
\begin{aligned}
& k\left(\left\|x_{i}-x_{j}\right\|, r\right)=\frac{1}{h \sqrt{2 \pi}} \exp \left(-\frac{\left(\left\|x_{i}-x_{j}\right\|-r\right)^{2}}{2 h^{2}}\right) \\
& g(r) \text { is estimated by: } \\
& \hat{g}(r)=\frac{1}{2 \pi r \widehat{\lambda^{2}}} \sum_{i} \sum_{j \neq i} k\left(\left\|x_{i}-x_{j}\right\|, r\right) c(i, j)
\end{aligned}
$$

where $c(i, j)$ is an edge-effect correction depending on both points. ${ }^{5}$ When a point is close to the boundary of the area under study, some of its neighbors are not observed because no data is available outside the area. Since the number of neighbors is underestimated for points located near the border of the domain, a statistical bias appears if no edge-effect correction is applied. For example, Marcon and Puech (2003), working on French data, showed that a severe bias existed in the distribution of manufacturing plants. A variety of edge-effect corrections are conceivable and have been proposed for relatively simple shapes (see Goreaud and Pélissier, 1999). However, these corrections are intractable for actual geographical units, such as countries or regions (Law et al., 2009). This has compelled authors to work on simple shapes to analyze the geographic distribution of economic activities (see Rowlingson and Diggle, 1993; Feser and Sweeney, 2000, among others). Ohser (1983) developed complex corrections for any polygonal window. They are implemented in the spatstat package for R (Baddeley and Turner, 2005; R Core Team, 2016), including the possibility to approximate any window by a raster image, as introduced by Wiegand et al. (1999).

$g$ is a topographic (restricted to homogeneous space), density function. It cannot be weighted.

\footnotetext{
${ }^{3}$ In the remainder, $x_{i}$ will designate reference points, $x_{j}$ their neighbors.

${ }^{4}$ To avoid any confusion in the paper, estimators will systematically be written with hats.

${ }^{5}$ Note that the unbiased estimator of $\lambda^{2}$ is $n(n-1) / A^{2}$ (Stoyan and Stoyan, 2000) where $n$ is the number of points and $A$ the surface of the study area.
} 


\subsection{The $K$ function}

Ripley $(1976,1977)$ summed $g$ on a range of distances from 0 to $r$ to define the $K$ function: $K(r)=\int_{0}^{r} g(\rho) 2 \pi \rho d \rho$.

If the point process is homogeneous and independent (i.e. it is a homogeneous Poisson process) the spatial pattern is called complete spatial randomness (CSR). In concrete terms, the CSR hypothesis means that all the points of the distribution have the same probability of being located anywhere on the territory: the density is constant everywhere in the domain. A complete spatial random distribution thus corresponds to a random distribution of independent points (no attraction or repulsion between points of the distribution under study). In the case of CSR, Ripley proved that the $K$ function reaches its benchmark: $K(r)=\pi r^{2}$. If $K(r)$ is greater than $\pi r^{2}$, then more points are found within a radius of $r$ from each point. The point process is said to be attractive and spatial concentration is detected. Values of $K(r)$ below $\pi r^{2}$ indicate that points repulse each other up to distance $r$ (dispersion).

As $K(r)$ is not easy to plot and $\pi r^{2}$ is not an easy-to-compare reference, Besag (1977) proposed to transform it into $L(r)=\sqrt{K(r) / \pi}$ so that its benchmark value is $r$, i.e. a straight line. It has often been plotted as $L(r)-r$ in the literature (Pélissier, 1998, for example) making its benchmark value 0 . The notation has gradually shifted and has become $L(r)=\sqrt{K(r) / \pi}-r$ (Goreaud and Pélissier, 1999, for example). Some authors define it as $H(r)$ (Kiskowski et al., 2009). $L(r)$ can be interpreted as a distance (Marcon and Puech, 2003): $L(r)=l$ means that as many neighbors are found around reference points up to distance $r$ as would be expected at distance $r+l$ if neighbor points are distributed independently of reference points. We believe that $K(r) / \pi r^{2}$ is a better normalization because it becomes a location quotient: the density of neighbors around reference points divided by the density of neighbors anywhere. Note that the normalized benchmark value is 1 .

Estimation of $K$ is done by counting neighbors up to $r$ and is defined as:

$$
\hat{K}(r)=\frac{1}{A \widehat{\lambda^{2}}} \sum_{i} \sum_{j \neq i} \mathbf{1}\left(\left\|x_{i}-x_{j}\right\| \leq r\right) c(i, j)
$$

$\mathbf{1}\left(\left\|x_{i}-x_{j}\right\| \leq r\right)$ is the indicator function equal to 1 if the distance between $x_{i}$ and $x_{j}$ is less than $r, 0$ else. $A$ is the surface area under study. Like $\hat{g}, \hat{K}$ suffers edge effects.

$K$ is the cumulative function of $g$ : it provides information up to a given distance while $g$ provides information at a distance. By analogy to probability density, the latter type of functions are called density functions below, although few of them are actually normalized so that they sum to 1 .

With $K$ intertype functions, neighbors of a particular type (Lotwick and Silverman, 1982) are counted around points of another type. The null hypothesis may be that points are labeled randomly or that point locations are independent. It must be chosen with care to avoid erroneous results (Arbia et al., 2008; Goreaud and Pélissier, 2003):

- random labeling is appropriate when locations are given, types are chosen. More precisely, in this case we assume that locations of points are given and that type-labeling is independent of the position of points. The hypothesis of random labeling is accurate for the location of shops in a city.

- population independence is the right hypothesis when points can be set anywhere, but not independently of other points of the same type. A good example of this hypothesis is trickier to find. One possible example could be interactions between two types of sellers on a beach (ice creams and sun hats, for instance) where each of them has its own spatial structure.

Empirical applications of Ripley's $K$ and Besag's $L$ functions to assess the geographical concentration of economic activities are limited. Some authors aim to detect the location patterns of subsectors of manufacturing industries (Marcon and Puech, 2003) or services (Ó hUallacháin and Leslie, 2007). Others depict specific location patterns of industrial production by focusing on one characteristic: plants size (Arbia, 1989) or the technology used (Barff, 1987), for example. However, these functions have two main limitations in the field of spatial economics preventing them from becoming wide used. The first one is related to the CSR hypothesis. The constant density benchmark is very strong for evaluating of the spatial distribution of activities and considerably limits the usefulness 
of the $K$ and $L$ functions in the field of spatial economics. To give some intuitive examples for the location of plants (we will come back to that point in the discussion section), this implies that there are no lakes or other locations where no buildings are permitted etc. The second important limitation is that the number of employees in the plants can not be taken into account: points can not be weighted. From an economic point of view it is hardly convincing if we aim to explain the agglomeration forces at work. This second limitation can be solved, however, by introducing a new function presented in the next subsection.

$K$ is a topographic (restricted to homogeneous space), cumulative function. It cannot be weighted.

\subsection{The $K_{m m}$ function}

The $K_{m m}$ function was introduced by Penttinen et al. (Penttinen, 2006; Penttinen et al., 1992). It generalizes Ripley's $K$ function by associating quantitative marks $w\left(x_{i}\right)$ to points that can be used as weights. By considering the spatial distribution of firms, a classical weight for the entities is the number of employees. However, other weights are possible, such as value-added per establishment.

The $K_{m m}$ function can be understood as a $K$ function computed on a data set where every point is associated with a mark (its weight in our case). The mark of the point $i$ is denoted $w\left(x_{i}\right)$ and that of point $j$ is $w\left(x_{j}\right)$. Its estimator is:

$$
\begin{aligned}
\hat{K}_{m m}(r) & =\frac{1}{A \widehat{\lambda^{2}} \overline{w^{2}}} \sum_{i} \sum_{j \neq i} \mathbf{1}\left(\left\|x_{i}-x_{j}\right\| \leq r\right) w\left(x_{i}\right) w\left(x_{j}\right) c(i, j) \\
& =\frac{A}{n(n-1) \overline{w^{2}}} \sum_{i} \sum_{j \neq i} \mathbf{1}\left(\left\|x_{i}-x_{j}\right\| \leq r\right) w\left(x_{i}\right) w\left(x_{j}\right) c(i, j)
\end{aligned}
$$

$\overline{w^{2}}$ is the average squared point weight, equal to $\frac{\sum_{i} \sum_{j \neq i} w\left(x_{i}\right) w\left(x_{j}\right)}{n(n-1)}$.

$\hat{K}_{m m}(r)$ was not normalized by $n(n-1) \overline{w^{2}}$ by Penttinen et al. (1992) who transformed it into $L_{m m}(r)=\sqrt{\frac{K_{m m}(r)}{\pi W^{2}}}$ where $W$ is the total weight of points. Studying the spatial concentration of plants, Giuliani et al. (2014) divided $\hat{K}_{m m}(r)$ by $W^{2}$ (we estimated it by $n(n-1) \overline{w^{2}}$ ) so that $\hat{K}_{m m}(r)$ has the same properties as $\hat{K}(r)$. We follow them and Illian et al. (2008), section 5.3.3. The benchmark value is reached if the points and marks are independent: $K_{m m}=\pi r^{2}$ and $L_{m m}=0$.

Note that the derivative of $K_{m m}$, that is to say the weighted equivalent of $g$ denoted $g_{m m}$, was first introduced by Stoyan and Ohser $(1984,1985)$ but not used in empirical applications.

In spatial economics, only one application of the $K_{m m}$ function can yet be found to date in Giuliani et al. (2014). In a few words, over the same period of observation (1996-2004), the $K_{m m}$ function detected agglomeration of high and high-medium technology manufacturing firms in the Milan's area (Italy), whereas no significant results appeared for these industries in the area of Turin.

$K_{m m}$ is a topographic (restricted to homogeneous space), cumulative function. It can be weighted.

\subsection{The $D$ function}

Ripley's $K$, Besag's $L$ and Penttinen et al.'s $K_{m m}$ functions consider space as homogeneous (as defined in section 2.1). To deal with non-stationary point patterns, Diggle and Chetwynd (1991) introduced the $D$ function, equal to the difference between two $K$ functions: that of the points of interest, called cases, and that of other points, called controls: $D=K_{c}-K_{0}$. The authors show that under the null hypothesis $K_{c}=K_{0}$. Both also equal the intertype function of cases and controls $K_{c, 0}$. When not zero, $D$ cannot be interpreted and is limited to tests. We introduce here another version of $D$ (previously advocated by Arbia et al., 2008), which we will denote $D_{i}$ :

$$
D_{i}=K_{c}-K_{c, 0}
$$

It also equals to 0 under the null hypothesis and can be used exactly like $D$. Its advantage compared to the $D$ of Diggle and Chetwynd is that it compares two $K$ functions computed around the same points (the cases). Thus, $D_{i} / \pi r^{2}$ is the difference between two location quotients: that of the cases around themselves and that of the cases 
around the controls. Finally, note that the $D$ function is also called the $K D$ function by Waller (2010), certainly due to the use of Ripley's $K$ function in the definition of the $D$ function.

From a statistical point of view, three important limitations of the $D$ function can be given. Firstly, values are not comparable on the same plot of the $D$ function. The excess number of points does not have the same signification at small distances as it does at greater ones (the expected number of points is greater at large distances). Secondly, over the same distances, values of two $D$ plots are not comparable if the controls are not the same. Changing the reference points (the controls) implies a change in the benchmark distribution: comparisons are then unfounded whatever the distance. Thirdly, the $D$ function results from a difference between two $K$ functions and therefore points can not be weighted.

This function was originally used by Diggle and Chetwynd to detect the spatial concentration of rare diseases. Despite the three limitations cited above, numerous applications of the $D$ function can be found in the field of spatial economics. In a pioneering empirical study, using the $D$ function, Sweeney and Feser (1998) showed that plants size matters in the measuring agglomeration of manufacturing firms in North Carolina (medium-sized plants show greater levels of spatial concentration). Marcon and Puech (2003) evaluated the spatial distribution of manufacturing firms in France and provided some comparisons with the results obtained using Ripley's original $K$ function on the same data. Other studies have investigated more sophisticated questions, such as a possible greater degree of spatial concentration for firms that are linked (Feser and Sweeney, 2000) or the existence of differences in the geographic concentration of patents according to the sector considered (Arbia et al., 2008).

$D$ is a topographic, cumulative function. It cannot be weighted.

\subsection{The $g_{\text {inhom }}$ and $K_{\text {inhom }}$ functions}

The $K$ function cannot be estimated from data if the point process is not stationary. Baddeley et al. (2000) derived the inhomogeneous version of $K$ called $K_{\text {inhom }}$, equal to the integral of $g_{\text {inhom }}$ and centered on $\pi r^{2}$ under the assumption of independence of points. It has been little used in economics (but see Arbia et al., 2009, 2012) because it requires the intensity of the point process to be estimated by kernel methods. If the kernel's bandwidth is very small, intensity is highly variable and independence is found, while a wide kernel results in more stationarity and dependence. In other words, the results are highly dependent on the arbitrary choice of the estimation kernel bandwidth (Diggle et al., 2007). If it is not guided by additional knowledge supporting it, results may be arbitrary.

Intuitively, $K_{\text {inhom }}$ counts the average number of neighbors on disks centered on the reference points, following $K$, but does not calculate the average the same way. The average value of the indicator function is weighted by the reciprocal of the intensity of the point process around each point, denoted $\lambda\left(x_{i}\right)$ for point $i$ and $\lambda\left(x_{j}\right)$ for point $j$. $\lambda\left(x_{i}\right)$ is defined as follow. Denote $\mathrm{d} x_{i}$ the elementary area around point $x_{i}, N\left(\mathrm{~d} x_{i}\right)$ the random number of points in $\mathrm{d} x_{i}$ and $\mathbf{E}\left(N\left(\mathrm{~d} x_{i}\right)\right)$ its expectation. Then, $\lambda\left(x_{i}\right)=\lim _{\mathrm{d} x_{i} \rightarrow 0} \frac{\mathbf{E}\left(N\left(\mathrm{~d} x_{i}\right)\right)}{\mathrm{d} x_{i}}$

$K_{\text {inhom }}$ is estimated by:

$$
\hat{K}_{\text {inhom }}(r)=\frac{1}{A} \sum_{i} \sum_{j \neq i} \frac{\mathbf{1}\left(\left\|x_{i}-x_{j}\right\| \leq r\right) c(i, j)}{\widehat{\lambda}\left(x_{i}\right) \hat{\lambda}\left(x_{j}\right)}
$$

$\widehat{\lambda}\left(x_{i}\right)$ and $\widehat{\lambda}\left(x_{j}\right)$ are the local density of points around $x_{i}$ and $x_{j}$, obtained by bi-dimensional kernel methods (Diggle, 1985). They are the estimators of the point process intensity. $g_{\text {inhom }}$ 's estimator can be found in Law et al. (2009):

$$
\hat{g}_{\text {inhom }}(r)=\frac{1}{2 \pi r} \sum_{i} \sum_{j \neq i} \frac{k\left(\left\|x_{i}-x_{j}\right\|, r\right) c(i, j)}{\widehat{\lambda}\left(x_{i}\right) \widehat{\lambda}\left(x_{j}\right)}
$$

In the spirit of Besag's $L$ function, the $L_{\text {inhom }}$ function is also proposed Arbia et al. (2012) and is estimated by:

$$
\hat{L}_{\text {inhom }}(r)=\sqrt{\frac{\hat{K}_{\text {inhom }}}{\pi}}
$$


The weighting of $g_{\text {inhom }}$ and $K_{\text {inhom }}$ may be clarified by defining $w\left(x_{i}\right)=\lambda / \lambda\left(x_{i}\right)$, i.e. the normalized reciprocal of the local intensity. If the intensity of the point process around point $x_{i}$ is greater than the average intensity $\lambda$, point $x_{i}$ receives a weight lower than 1 , and conversely. In the same way, we also define the normalized reciprocal of the local intensity around $x_{j}: w\left(x_{j}\right)=\lambda / \lambda\left(x_{j}\right)$. Then, the estimators of $g_{\text {inhom }}$ and $K_{\text {inhom }}$ write as those of $g_{m m}$ and $K_{m m}$,

with the average square weight $\overline{w^{2}}$ set to 1 , so that $K_{\text {inhom }}$ simplifies to $K$ for homogenous processes (Baddeley et al., 2000). Thus, $K_{\text {inhom }}$ and $K_{m m}$ are formally similar, but the meaning of weights is very different: in $K_{m m}$, weights are an attribute of points (such as the number of employees of firms) whilst they are a feature of space (a function of the local intensity of the point process) in $K_{\text {inhom }}$.

Despite the great qualities of the $K_{\text {inhom }}$ function, its applications are still scarce in economics. To the best of our knowledge, the first paper that introduced this function in our field was by Bonneu (2007). In this paper, he analyzed the distribution of fire department emergencies in the area of Toulouse (France) in 2004. Another application was recently proposed by Arbia et al. (2012). They provide an evaluation of the spatial distribution of five sectors of the high-tech industry in 2001 in Milan (Italy). The authors justified the use of the $L_{\text {inhom }}$ function for its potential in a dynamic context, although even if they did not deal with space-time analysis in their paper.

$g_{\text {inhom }}$ and $K_{\text {inhom }}$ are topographic, density and cumulative functions. They cannot address point weights.

\subsection{The $K_{d}$ function}

Duranton and Overman (2005)'s $K_{d}$ is the probability density function to find a point's neighbor at a given distance. It counts and averages the number of point pairs at each distance, and smooths the results to obtain a continuous function that is normalized to sum to 1 . Values of $K_{d}$ are compared to the confidence interval of the null hypothesis that points are randomly placed on their actual location set. A variant of $K_{d}$ named $K^{e m p}$ (also proposed by Duranton and Overman, 2005) allows points to be weighted, counting employees in firms. Actually, $K_{d}$ and $K^{e m p}$ are the densities of neighbor points and neighbor employees around reference points, according to the definition of their estimators by their authors. Duranton and Overman's kernel function $f$ in their definition of $\hat{K}_{d}(r)$ is actually not the previously defined kernel function but $k\left(\left\|x_{i}-x_{j}\right\|, r\right) h$. We have:

$$
\begin{aligned}
& \hat{K}_{d}(r)=\frac{1}{n(n-1)} \sum_{i} \sum_{j \neq i} k\left(\left\|x_{i}-x_{j}\right\|, r\right) \\
& \hat{K}^{e m p}(r)=\frac{1}{n(n-1) \overline{w^{2}}} \sum_{i} \sum_{j \neq i} w\left(x_{i}\right) w\left(x_{j}\right) k\left(\left\|x_{i}-x_{j}\right\|, r\right)
\end{aligned}
$$

There is no benchmark for $K_{d}$ and $K^{e m p}$ under the null hypothesis of independence between point locations. For each distance, the benchmark is obtained by simulations of the null hypothesis: it is the center of the confidence interval. The null hypothesis is built by redistributing the reference points over all points locations. In other words, $K_{d}$ is applied to a single type of points (e.g. one manufacturing sector) among a wider point pattern (e.g. the whole manufacturing industry).

The $K_{d}$ function is now considered one of the leading functions in spatial economics. In consequence, Duranton and Overman's methodology has been widely applied in our field since their seminal paper. In this paper, they broadly depicted the advantages of the $K_{d}$ function on an exhaustive dataset of UK manufacturing plants in 1996 at the four-digit sectoral level. In a later study, Duranton and Overman (2008) paved the way for future research by studying various pertinent economic questions on location patterns: plant entries and exits, affiliated and nonaffiliated plants, domestic and foreign plants etc. Then their methodology has been applied to various countries (see Klier and McMillen, 2008, among others) and extended to empirical studies in services (Nakajima et al., 2012; Barlet et al., 2013; Koh and Riedel, 2014). Behrens and Bougna (2015) preferred to use the $K_{d}$ function to depict change in the spatial distribution of manufacturing activities in Canada from 2001 to 2009. They also tested the importance of the level of sectoral aggregation as Fratesi (2008) did for the distribution of the pharmaceutical and optical-photographic sectors in Great-Britain. 
Three recent interesting contributions have been made. The first one is proposed by Kerr and Kominers (2015) who use the $K_{d}$ function to understand the spatial cluster patterns. The second is done by Behrens and Bougna (2015) who introduced the cumulative versions of the weighted and unweighted $K_{d}$ function $\left(i . e . \int_{0}^{r} K_{d}(r) \mathrm{d} r\right)$. This development seems to be motivated by the difficulties of interpreting $K_{d}$ results. These new but unnamed cumulative functions of $K_{d}$ provide the proportion of plant pairs located less than a distance $r$ apart. Some comparisons with the $K_{d}$ function or Ellison and Glaeser's index (1997) were given. The authors did not provide the confidence interval of the null hypothesis, which could have been calculated the same way as that of $K_{d}$. Finally, Marcon and Puech (2010, 2015) provided theoretical and empirical comparisons of the $K_{d}$ results with other distance-based methods (like the $D$ function presented before).

$K_{d}$ is an absolute, density function. $K^{e m p}$ is its weighted version.

\subsection{The $M$ and $m$ functions}

Marcon and Puech's (2010) $M$ function is a cumulative function that gives the relative frequency of neighbors of a chosen type up to each distance, compared to the same ratio in the whole area under study. The neighbors of the chosen type (say, a sector of activity) are denoted $x_{j}^{c}$, while $x_{j}$ is for any neighbor whatever its type. $M$ is estimated by:

$$
\hat{M}(r)=\sum_{i} \frac{\sum_{j \neq i} \mathbf{1}\left(\left\|x_{i}-x_{j}^{c}\right\| \leq r\right) w\left(x_{j}^{c}\right)}{\sum_{j \neq i} \mathbf{1}\left(\left\|x_{i}-x_{j}\right\| \leq r\right) w\left(x_{j}\right)} / \sum_{i} \frac{W_{c}-w\left(x_{i}\right)}{W-w\left(x_{i}\right)}
$$

$W_{c}$ is the total weight of the points $x_{j}^{c}$ and $W$ the total weight of all points of the sample. The denominator is slightly different in the intertype function, $\sum_{i}\left\{W_{c} /\left[W-w\left(x_{i}\right)\right]\right\}$, avoiding a small bias. For any distance, the benchmark value is thus $1 .^{6}$

Some empirical applications of the $M$ function can be found in economics. For instance Jensen and Michel (2011) developed the unweighted version of the $M$ function to gauge the spatial pattern of shops in the Lyon area in France. Marcon and Puech (2015) compare the empirical results obtained with the $M$ function to other distance-based methods ( $D$ and $K_{d}$ ) to show the limitation of each measure. Finally, although Marcon and Puech (2010) showed that $M$ and $K_{d}$ are complements rather than substitutes, applications using the $M$ function in economics are undoubtedly less numerous than those using $K_{d}$. However, it is interesting to note that the $M$ function has been rapidly transferred to other scientific fields, such as geography (Deurloo and De Vos, 2008), ecology (Marcon et al., 2012), biology (Fernandez-Gonzalez et al., 2005) or seismology (Nissi et al., 2013).

In a recent paper Lang et al. (2015) proposed the $m$ function: a density function version of $M$. By keeping the same notations, the estimator of $m$ is:

$$
\hat{m}(r)=\sum_{i} \frac{\sum_{j \neq i} k\left(\left\|x_{i}-x_{j}^{c}\right\|, r\right) w\left(x_{j}^{c}\right)}{\sum_{j \neq i} k\left(\left\|x_{i}-x_{j}\right\|, r\right) w\left(x_{j}\right)} / \sum_{i} \frac{W_{c}-w\left(x_{i}\right)}{W-w\left(x_{i}\right)}
$$

As in Duranton and Overman (2005), a Gaussian kernel of optimal bandwidth was applied according to Silverman (1986). The numerator is the local ratio: the relative weight of neighbors of interest at distance $r$ from all reference points and averaged over all reference points. The denominator corresponds to the global ratio: it is defined in the same way as the local ratio, but over the whole data set. 1 is the benchmark value for any distance $r$ and the generation of a confidence interval of the null hypothesis with Monte-Carlo simulations provide the significance of the $m$ estimates.

Note the similarities between $m$ and the cumulative $M$ function, but that the local ratio is defined at distance $r$ and not up to it for the $m$ function.

$m$ and $M$ are relative, density and cumulative functions. They can be weighted.

\footnotetext{
${ }^{6}$ In extreme cases, if the industrial concentration (in the sense of Ellison and Glaeser, 1997) is too high, for every radius $r$, the benchmark value would be the center of the confidence interval.
} 


\section{A typology of distance-based methods}

In what follows, we shall prove that all these functions can be built empirically following the same five steps. First, neighbors are counted around each point at or within a distance $r$; sometimes weights are summed instead. Second, an average number of neighbors $\bar{n}(r)$ is calculated. Third, $\bar{n}(r)$ is divided by a local reference $z(r)$. In accordance with the typology of Brülhart and Traeger (2005) we shall use the following vocabulary:

- Topographic measures use space as their benchmark: the number of neighbors is divided by the area of a ring or a disk $\left(2 \pi r \mathrm{~d} r\right.$ or $\left.\pi r^{2}\right)$.

- Relative measures divide the number of neighbors of interest by that of all neighbors.

- Absolute measures do not have any benchmark.

Fourth, $\bar{n}(r) / z(r)$ is compared to the value it has on the whole domain, $\bar{n}_{0} / z_{0}$, to normalize the function. Fifth and last, the significance of the values of the functions at several distances is generally tested against a null hypothesis by Monte-Carlo simulations of the appropriate counterfactual. These five steps are detailed below.

\subsection{Step 1: a number of neighbors $n\left(x_{i}, r\right)$}

The first step consists in counting neighbors of each point, at distance $r$ (on the circle of radius $r$ ) or up to distance $r$ (in the circle of radius $r$ ). The first option defines density functions: $g, g_{\text {inhom }}, K^{e m p}, K_{d}$ and $m$. The second defines cumulative functions: $K, K_{\text {inhom }}, K_{m m}, D_{i}$, the cumulative of $K^{e m p}$, the cumulative of $K_{d}$, and $M . M, K^{e m p}$ and $K_{m m}$ attribute a weight to points, such as the plants' number of employees. This raw number of neighbors of the point $i$ at a distance $r$ or up to $r$ is denoted $n\left(x_{i}, r\right) . g_{\text {inhom }}$ and $K_{\text {inhom }}$ do not just count 1 for each point but give them a weight that is inversely proportional to the local density of points: more neighbors are expected where more points are located and these portions of space must not be overweighted.

Table 1 summarizes the way neighbors are counted around reference points for each function. Reference points (circle centers) are denoted $x_{i}$, their neighbors are $x_{j}$. The point types may be identical or not, defining intertype functions in the latter case. By construction, $M$ focuses on one special type of neighbor points denoted $x_{j}^{c}$ to compare their distribution to that of all neighbors denoted $x_{j}$ at step 3. $w\left(x_{j}\right)$ is the weight of point $x_{j} . \widehat{\lambda}\left(x_{j}\right)$ is the density of points around $x_{j}$. It is the estimator of $\lambda\left(x_{j}\right)$, the intensity of the point process. As we have already noted, we use hats in equations for estimators to avoid any confusion: $K, g$ have a mathematical definition relying on the point process they are used to characterize and are estimated from the data. We also define $K_{d}$ and $M$ in this way in this paper. $k\left(\left\|x_{i}-x_{j}\right\|, r\right)$ is some kernel function able to evaluate the number of neighbors at distance $r . c(i, j)$ is some edge-effect correction depending on both points.

\subsection{Step 2: Computing an average number of neighbors}

The value obtained around each point following table 1 is then averaged for all reference points. In topographic, inhomogeneous measures, the weight of each point is inversely proportional to the intensity of the process around it so that space is sampled uniformly. All points have the same weight in $K_{d}$ and $M$.

Table 2 summarizes the way the average number of neighbors is calculated. $\bar{n}(r)$ is the average number of neighbors. $n$ is the total number of reference points (the centers of circles).

\subsection{Step 3: Defining a local reference and identifying the nature of the measure}

These numbers of neighbors are then divided by a local reference $z(r)$, table 3 . This step determines the nature of the measure:

- if $z(r)$ is a measure of space, the function is topographic,

- if it is a number of neighbors, the function is relative,

- if there is no benchmark, the function is absolute. 
Table 1. Estimating the number of neighbors, $n\left(x_{i}, r\right)$

\begin{tabular}{|c|c|c|}
\hline Function & Neighbors around $x_{i}$ & Observations \\
\hline$\widehat{K}(r) /\left(\pi r^{2}\right)$ & $n\left(x_{i}, r\right)=\sum_{j \neq i} \mathbf{1}\left(\left\|x_{i}-x_{j}\right\| \leq r\right) c(i, j)$ & $\begin{array}{l}\text { The number of neighbors is counted and } \\
\text { corrected from edge effects. }\end{array}$ \\
\hline $\begin{array}{l}\widehat{K}_{m m}(r) /\left(\pi r^{2}\right) \\
\widehat{K}_{\text {inhom }}(r) /\left(\pi r^{2}\right)\end{array}$ & $n\left(x_{i}, r\right)=\sum_{j \neq i} \mathbf{1}\left(\left\|x_{i}-x_{j}\right\| \leq r\right) w\left(x_{j}\right) c(i, j)$ & $\begin{array}{l}\text { As } K \text { above, but each neighbor counts } \\
\text { for its weight. In } K_{\text {inhom, }} \text {, weights are } \\
\lambda / \widehat{\lambda}\left(x_{j}\right) \text {. }\end{array}$ \\
\hline$\widehat{g}(r)$ & $n\left(x_{i}, r\right)=\sum_{j \neq i} k\left(\left\|x_{i}-x_{j}\right\|, r\right) c(i, j)$ & $\begin{array}{l}\text { As } K \text { above, but the neighbors are counted } \\
\text { at distance } r \text {. }\end{array}$ \\
\hline$\widehat{g}_{\text {inhom }}(r)$ & $n\left(x_{i}, r\right)=\sum_{j \neq i} k\left(\left\|x_{i}-x_{j}\right\|, r\right) w\left(x_{j}\right) c(i, j)$ & $\begin{array}{l}\text { As } g \text { above, but each neighbor counts for } \\
\text { the inverse of the density around it. In } \\
g_{\text {inhom }} \text {, weights are } \lambda / \hat{\lambda}\left(x_{j}\right) \text {. }\end{array}$ \\
\hline$\widehat{K}_{d}(r)$ & $n\left(x_{i}, r\right)=\sum_{j \neq i} k\left(\left\|x_{i}-x_{j}\right\|, r\right)$ & $\begin{array}{l}\text { As } g \text { above, without edge-effect correc- } \\
\text { tion. }\end{array}$ \\
\hline$\widehat{K}^{e m p}(r)$ & $n\left(x_{i}, r\right)=\sum_{j \neq i} k\left(\left\|x_{i}-x_{j}\right\|, r\right) w\left(x_{j}\right)$ & $\begin{array}{l}\text { As } K_{d} \text { above, but each neighbor counts for } \\
\text { its weight. }\end{array}$ \\
\hline$\widehat{M}(r)$ & $n\left(x_{i}, r\right)=\sum_{j \neq i} \mathbf{1}\left(\left\|x_{i}-x_{j}^{c}\right\| \leq r\right) w\left(x_{j}^{c}\right)$ & $\begin{array}{l}\text { Each neighbor of the type of interest } \\
\text { counts for its weight. }\end{array}$ \\
\hline$\widehat{m}(r)$ & $n\left(x_{i}, r\right)=\sum_{j \neq i} k\left(\left\|x_{i}-x_{j}^{c}\right\|, r\right) w\left(x_{j}^{c}\right)$ & $\begin{array}{l}\text { As } M \text { above, but the neighbors are } \\
\text { counted at distance } r \text {. }\end{array}$ \\
\hline
\end{tabular}

Table 2. Average number of neighbors, $\bar{n}(r)$

\section{Function}

$$
\widehat{K}(r) /\left(\pi r^{2}\right)
$$$$
\widehat{g}(r)
$$

$\widehat{K}_{d}(r)$

$\widehat{M}(r)$

$\widehat{m}(r)$

$$
\begin{array}{ll}
\widehat{K}_{m m}(r) /\left(\pi r^{2}\right) & n(r)=\frac{1}{n w^{2}} \sum_{i} w\left(x_{i}\right) n\left(x_{i}, r\right) \\
\widehat{K}^{\text {emp }}(r) & \\
\widehat{K}_{\text {inhom }}(r) /\left(\pi r^{2}\right) & \\
\widehat{g}_{\text {inhom }}(r) &
\end{array}
$$

\section{Observations}

The number of neighbors around each point is not weighted. $n$ is the number of circle centers, limited to the number of points of the sector of interest in $M$ and $m$.

The average is weighted $\left(\overline{w^{2}}\right.$ is the average squared weight). In $g_{\text {inhom }}$ dans $K_{\text {inhom }}$, weights are $\lambda / \widehat{\lambda}\left(x_{i}\right)$ and $\overline{w^{2}}=1$. 
Table 3. Defining a local reference $z(r)$ and identifying the nature of the measure

\begin{tabular}{|c|c|c|}
\hline Function & Local reference & Observations \\
\hline $\begin{array}{l}\widehat{K}(r) /\left(\pi r^{2}\right) \\
\widehat{K}_{\text {inhom }}(r) /\left(\pi r^{2}\right) \\
\widehat{K}_{m m}(r) /\left(\pi r^{2}\right)\end{array}$ & $z(r)=\pi r^{2}$ & The local reference is the area of a disk. \\
\hline $\begin{array}{l}\widehat{g}(r) \\
\widehat{g}_{\text {inhom }}(r)\end{array}$ & $z(r)=2 \pi r$ & As $K$ above, but the measure is the length of a ring. \\
\hline $\begin{array}{l}\widehat{K}_{d}(r) \\
\widehat{K}^{\text {emp }}(r)\end{array}$ & $z(r)=1$ & $\begin{array}{l}K_{d} \text { is not compared to anything. It is an absolute mea- } \\
\text { sure. }\end{array}$ \\
\hline$\widehat{M}(r)$ & $z(r)=\frac{1}{n} \sum_{j \neq i} \mathbf{1}\left(\left\|x_{i}-x_{j}\right\| \leq r\right) w\left(x_{j}\right)$ & $\begin{array}{l}\text { The number of neighbors of the type of interest is com- } \\
\text { pared to the number of neighbors of all types. }\end{array}$ \\
\hline$\widehat{m}(r)$ & $z(r)=\frac{1}{n} \sum_{j \neq i} k\left(\left\|x_{i}-x_{j}\right\|, r\right) w\left(x_{j}\right)$ & As $M$ above. \\
\hline
\end{tabular}

In relative functions, "all" neighbors usually include those of the reference type. They may be excluded (Diggle et al., 2007; Marcon et al., 2012), for instance if the sampling design is such that all points of interest (the cases), but only a sample of the benchmark distribution (the controls) are recorded. Then, the whole distribution of points is better represented by the controls alone.

\subsection{Step 4: Defining a global reference (a normalizing constant)}

Normalization comes next (table 4$) . \bar{n}(r) / z(r)$ is divided by its normalizing constant $\bar{n}_{0} / z_{0}$. The latter ratio can be understood as the value of the former with $r$ large enough for all points to be neighbors of each other. Then, possible spatial structure does not matter: $\bar{n}_{0}$ counts all points except the center of the circle, $z_{0}$ is the total area (topographic measures) or the total number of points (relative measures) or 1 (absolute measures).

All measures in table 4 are location quotients except for $K_{d}$ and $K^{\text {emp }}$ which are absolute measures.

\subsection{Last step: Null hypothesis}

We have obtained a value for each function at or within a distance $r$. Computation can be repeated for several values of $r$ to get each concentration measure as a function of distance.

How can the significance of the results be tested? In the vast majority of empirical studies, authors use MonteCarlo simulations to decide whether or not values obtained by the previous functions reject the null hypothesis tested.

Practically, thanks to Monte-Carlo simulations, a confidence interval of the results is proposed. In a general manner, Monte-Carlo simulations provide many values of the simulated function for each value of $r$. A proportion of them is eliminated according to the accepted risk level (often the greatest and the smallest $2.5 \%$ in order to obtain a 95\% confidence interval). The remaining values constitute the local (i.e. at $r$ ), also called pointwise, confidence interval of the null hypothesis. To test the significance of the results, for every value of $r$ the observed value of the function is compared to the local confidence interval. Lagache et al. (2013) developed a method to calculate the quantiles of $K(r)$ under the null hypotheses of CSR analytically, without any simulation. Duranton and Overman (2005) noted that repeating the same local test for all values of $r$ is not satisfactory as the resulting local confidence interval would be restrictive enough. This is confirmed by the findings of Loosmore and Ford (2006) and Loop and McClure (2015) who proved the inadequacy of the local test. Duranton and Overman (2005) proposed a 
Table 4. Global reference: the normalizing constant, $\bar{n}_{0} / z_{0}$

\begin{tabular}{|c|c|c|}
\hline Function & Global reference & Observations \\
\hline $\begin{array}{l}\widehat{K}(r) /\left(\pi r^{2}\right) \\
\widehat{g}(r) \\
\widehat{K}_{\text {inhom }}(r) /\left(\pi r^{2}\right) \\
\widehat{g}_{\text {inhom }}(r) \\
\widehat{K}_{\text {mm }}(r) /\left(\pi r^{2}\right)\end{array}$ & $\frac{\bar{n}_{0}}{z_{0}}=\frac{n-1}{A}$ & $\begin{array}{l}\text { The normalizing constant is the intensity of the point } \\
\text { process, evaluated by the total number of points minus } 1 \\
\text { (the circle center) divided by the area of the window. }\end{array}$ \\
\hline $\begin{array}{l}\widehat{K}_{d}(r) \\
\widehat{K}^{\text {emp }}(r)\end{array}$ & $\frac{\bar{n}_{0}}{z_{0}}=n-1$ & $\begin{array}{l}\text { Absolute measure. } z_{0}=1 . \bar{n}_{0} \text { is the total number of } \\
\text { points minus } 1 \text { (the point at the circle center). }\end{array}$ \\
\hline $\begin{array}{l}\widehat{M}(r) \\
\widehat{m}(r)\end{array}$ & $\frac{\bar{n}_{0}}{z_{0}}=\sum_{i} \frac{W_{c}-w\left(x_{i}\right)}{W-w\left(x_{i}\right)} \approx \frac{W_{c}}{W}$ & $\begin{array}{l}\text { The normalizing constant is calculated as } \bar{n}(r) / z(r) \\
\text { with } r \text { large enough for all points to be neighbors to } \\
\text { each other. } W_{c} \text { is is the total weight of points belonging } \\
\text { to the neighbor type, } W \text { the total weight of all points. }\end{array}$ \\
\hline
\end{tabular}

way to build a global (more conservative) test, followed by Marcon and Puech (2010). This global test has been justified theoretically by Myllymäki et al. (2015) who called it the "rank envelope test". Barlet et al. (2013) showed empirically that it is still not restrictive enough when the number of simulations is too small relatively to the number of points: its confidence level is overestimated so $K_{d}$ detects localization where there may not be any. The solution is to increase the number of simulations.

For every intratype function, technical explanations of the construction of the associated confidence interval are summarized in table 5 .

The null hypothesis of intratype topographic functions is that the point process is Poisson so the simulations are drawn from a Poisson process of the intensity estimated from the data. Bivariate topographic functions have two possible null hypotheses presented in section 2.2: random labeling and population independence. Random labeling is simulated by redistributing the point types randomly across points. Population independence is simulated by shifting the whole neighbor point type randomly relatively to the reference point type. Relative and absolute intratype functions rely on the random location null hypothesis first proposed by Duranton and Overman (2005). Since nothing is known about the geography of the area under study except for the actual location of points, the null hypothesis is simulated by redistributing the points (with their type and weight) across these locations. For intertype functions, random labeling is simulated by randomizing point types, keeping locations and weights unchanged whereas population independence keeps reference points unchanged and only randomizes the other point locations (Marcon et al., 2015). Note that they are the standard null hypotheses of the literature: they are not limitative. Any null hypothesis may be tested so long as it can be simulated, such as aggregative point processes to test by topographic functions (Ngo Bieng et al., 2011).

It can be noted that $K_{d}$ does not contain any reference to the overall distribution of points, as a relative measure would. Comparing $K_{d}$ to its simulated values (null hypothesis) provides a concentration test since the departure from randomness is detected from all occupied sites observed in the real distribution. The expectation of the value $K_{d}$ of the simulated distributions is simply the value of $K_{d}$ calculated on the whole point set since points of the type under study are redistributed on all actual locations with the same probability, but its variability is unknown so simulations cannot be avoided.

$M$ and $m$ allow the points to be given a weight, typically a number of employees. But it does not take into account the possible influence of the structure of point weights. However, the necessity of controlling for industrial concentration was highlighted by Ellison and Glaeser (1997). The answer is the following: the simulations are done 
Table 5. Simulation of the null hypothesis

Function Null Hypothesis

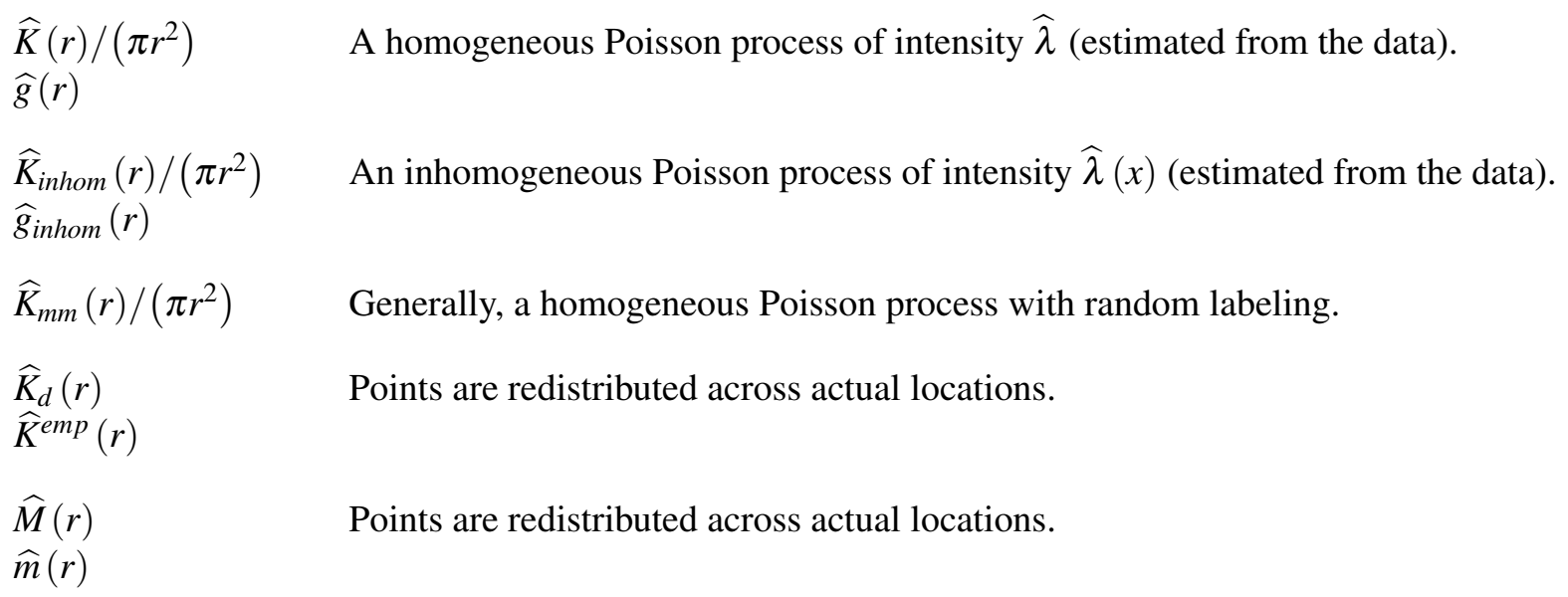

with the actual weights, so the weight structure is controlled for by the confidence interval of the null hypothesis, if not numerically.

The null hypothesis of $K_{m m}$ varies. Penttinen et al. (1992) inferred the unmarked point process model and used it to simulate points with a random permutation of marks. The null hypothesis was the independence of marks, given the point set structure. Practically, $K_{m m}$ can be used when the unmarked point process is a homogeneous Poisson, but with great difficulty in other situations. Giuliani et al. (2014) integrated both point locations and mark structure in a single model of localization, whose parameters cannot be inferred yet.

$K_{\text {inhom }}$ was employed by Diggle et al. (2007) and Arbia et al. (2012) in a case-control design: a number of points, the controls, were used to estimate the point process intensity (assuming they are approximately independently distributed), while the different pattern of cases was attributed to dependence. This approach is quite similar to that of $M$ where all points weight 1: estimating $\widehat{\lambda}\left(x_{j}\right)$ in table 1 for $K_{\text {inhom }}$ with a simple box kernel with bandwidth $r$ is not different from $z(r)$ in table 3 for $M$. The main difference (normalization apart) is that all reference points have the same weight in $M$, while each piece of space has in $K_{\text {inhom }}$ (table 2).

Goodness-of-fit (GoF) tests are an alternative explored by Heinrich (1991). They consist of calculating the discrepancy between an estimated function for the real data and its expectation under the null hypothesis: the value obtained is compared to its distribution under the null hypothesis. In the general case, neither the expectation nor the quantiles of the distribution are known so they must be obtained by Monte-Carlo simulations. Loosmore and Ford (2006) published a GoF test for the $K$ function in ecology. Marcon et al. (2012) proposed an application of the GoF test for the $M$ function in ecology too. Barlet et al. (2013) developed a GoF test for the $K_{d}$ function and proved it to be unbiased.

Global, analytical tests (providing a p-value to reject the null hypothesis erroneously, calculated from the data without simulations) are scarce. Jensen and Michel (2011) provided one for $K_{d}$ based on the exact calculation of the variance and one for the unweighted version of $M$. Lang and Marcon (2013) developed a test for $K$ against CSR in a square domain, Marcon et al. (2013) extended it to a rectangle domain and applied it to ecological data. A non-trivial advantage of analytical tests is saving the time involved in calculating of confidence intervals, which is quite long for large datasets. 
Table 6. Choice of the appropriate function to describe a point pattern structure

\begin{tabular}{lcccc}
\hline Function choice & $\begin{array}{c}\text { Topographic, } \\
\text { homogeneous }\end{array}$ & $\begin{array}{c}\text { Topographic, } \\
\text { inhomogeneous }\end{array}$ & Absolute & Relative \\
\hline Density functions & $g$ & $g_{\text {inhom }}$ & $K_{d}$ & $K^{\text {emp }}$ \\
\cline { 2 - 4 } Cumulative functions & $K$ & $K_{\text {inhom }}$ & Cumulative of $K_{d}$ & $M$ \\
& $K_{m m}$ & $D_{i}$ & Cumulative of $K^{\text {emp }}$ & Case-control $K_{\text {inhom }}$ \\
\hline
\end{tabular}

\section{Discussion}

The aim of the previous section was to propose a common framework for understanding the statistic construction of the most popular distance-based methods. In this section, we shall provide a discussion of those functions with the objective of addressing economic questions.

\subsection{Complementary functions for addressing multiple questions}

The spatial structures of economic activities can be tested or evaluated in many ways because they are related to different economic questions. In that sense, distance-based measures appear more complementary than competing functions because of the richness of the results they provide. In the previous section, we presented more than ten distance-based measures and we showed that every distance-based measure brings a particular description of the data. The use of several functions may bring complementary results on the spatial distribution analyzed and thus give a comprehensive analysis of the data. In each case, a significant departure from randomness can be detected but the analysis is not the same, so results on the underlying spatial structure obviously will not systematically converge. However the choice of the distance-based measure(s) retained for any analysis should be well-motivated to respond at best to the economic question raised. This choice is not obvious and we propose a typology of all functions to find the "good" distance-based measure(s) for any analysis. This typology (given in table 6) is constructed according to two important tools' properties:

- the nature of the spatial concentration studied (topographic, absolute or relative). This first choice is crucial to avoid any misinterpretation of the data (Marcon and Puech, 2015). For example, urbanization externalities may be addressed by topographic functions to relate topographic concentration and productivity (Ciccone and Hall, 1996), whilst externalities induced by co-agglomeration (Ellison et al., 2010) require relative measures.

- a cumulative or a local approach. Analyzing the surroundings of the plants "at" a given distance or "up to" a given distance is not the same thing. In the first case cumulative functions should be used, in the second case density functions should be preferred (Marcon and Puech, 2010).

Let us now explain in details our motivation for that first classification of distance-based methods.

Consider at first the criterion of the nature of the spatial concentration. As we underlined, distance-based methods that integrate physical space as their benchmark are topographic measures. Those which refer to another variable, usually the distribution of all points, are called relative measures. Researchers should carefully motivate the type of concentration that they want to use the appropriate function. It the question is "Is the proportion of the manufacturing plants of the sector of interest in the neighborhood greater or lower than expected by chance?" then relative concentration methods are appropriate but if it is "Is the density of neighbor manufacturing plants per unit area greater or lower than expected by chance?", this refers to topographic concentration. It is worth noting 
that "absolute concentration" is not an operational concept (Combes and Overman, 2004; Brülhart and Traeger, 2005). The local reference of topographic and relative functions corresponds to the hypothesis they test. In contrast, absolute measures include no benchmark. Thus, the null hypothesis defines the type of concentration to address: the random-location hypothesis supposes the distribution of the economic sector under study is that of the whole industry, i.e. a departure from it reveals relative concentration. Alternatively, the null hypothesis could be CSR to test topographic concentration. In summary, absolute functions are versatile since they allow testing both topographic and relative concentration, even though we are not aware of empirical studies using $K_{d}$ to test topographic concentration. Yet, the usual way to apply $K_{d}$ to test relative concentration is sometimes problematic. The fundamental issue is that an absolute measure necessarily has an implicit reference: with $K_{d}$, the number of neighbors are counted on a circle around each point, as in the $g$ function for instance. The characteristic inverted $\mathrm{U}$ shape of the $K_{d}$ function is determined, at the first order, by geometry: the length of the perimeter of the circle around each point, which hosts the neighbors, increases proportionally to $r$ until the edge effects start to dominate, when the circle is too wide and finally exceeds the size of the data set. The length of the perimeter of this circle is $g$ 's benchmark, defining it as a topographic function, whereas it is ignored by $K_{d}$. So $K_{d}$ finally compares, by difference, the (normalized) number of neighbors of the points of the type of interest to that of all points. This is very similar to what the $D$ function does: actually, $K_{d}$ compares the topographic concentration of the sector of interest to that of the whole economic activity instead of addressing relative concentration directly. Let us consider a real example to make things clearer. Marcon and Puech (2015) showed that in the metropolitan area of Lyon (France), gas stations are topographically dispersed: the $D$ function is negative up to the maximum distance studied, $3 \mathrm{~km}$. This means that the normalized (see table 4) average (see table 2) number of neighbor gas stations around a typical gas station is lower than the normalized average number of neighbor shops of all other sectors of activity. Yet, gas stations are relatively concentrated: the $M$ function shows that around a typical one, the proportion of gas stations among all neighbor shops is higher than the average proportion of gas stations in the whole area. Indeed, gas stations are located in low-density shopping areas: they are less topographically concentrated than the commercial activity in general, but in these areas, they are relatively concentrated: two measures reveal two aspects of the spatial structure of the sector of activity. $K_{d}$ detects significant dispersion, as a topographic measure. We acknowledge that generally, relative concentration and higher topographic concentration than the whole activity co-exist: the empirical consequences of this ambiguity of $K_{d}$ are limited.

Let us turn to the second criterion: the choice between a cumulative or a local approach. This is a recent open debate that concerns the respective advantages of probability density functions and cumulative measures (Wiegand and Moloney, 2004; Law et al., 2009; Marcon and Puech, 2010). Arguments are not repeated here (the reader should refer to the cited papers) because the choice depends on both the question analyzed and the availability of data. Marcon and Puech (2010) have shown that $K_{d}$ or $M$ are useful to evaluate the spatial distribution of activities and depending on the question raised, one may give clearer results than the other. $K_{d}$ provides more precise estimations than $M$ for gauging the local density of activities. Thus, $K_{d}$ should be preferred if the objective is the evaluation of local densities. $M$ assesses the global effect of the superposition of spatial structures better. As a consequence, if the question is "up to which distance do externalities matter?" then a cumulative function is more appropriate. A probability density function will answer "do externalities matter at a given distance?" in a more satisfactory way. As a consequence, $M$ and $K_{d}$ seem to be complements rather than substitutes.

To what extent may all the distance-based methods presented here properly address the measurement of agglomeration or dispersion of economic activities? This question is very important to satisfactorily gauge the economic forces at work (Ellison et al., 2010; Kerr and Kominers, 2015). An axiomatic approach can be proposed. In particular, in their seminal paper Duranton and Overman listed five important properties required for any statistical measure aiming to detect spatial structures in economics (Duranton and Overman, 2005, p.1079):

1. results should be comparable across industries;

2. the global agglomeration pattern should be taken into account; 
3. the measure should control for industrial concentration in the sense of Ellison and Glaeser (1997);

4. results should be unbiased across geographic scales (this is related to the MAUP issues);

5. test of the significance of the results should be provided

An overview of the typology provided in this paper shows that only four other functions $K_{d}, K_{\text {inhom }}, M$ and $m$ respect all five criteria above (noticeably because criterion 3 excludes homogeneous, topographic concentration). As we previously underlined, $K_{d}$ is certainly now the most employed distance-based method in the economic literature. However it appears that also $K_{\text {inhom }}, M$ and $m$ are good candidates to analyze spatial concentration in spatial economics.

A second possible criterion of decision should be of interest: researchers may be interested not only in detecting the spatial structures but also in quantifying agglomeration or dispersion results. Up to now, only a small number of the distance-based methods listed previously can be mobilized to reach that objective. If the question is how many times more neighbors are found at the chosen distance then only $g, g_{\text {inhom }}$ and $m$ are of interest. If the question is how many times more neighbors are found up to the chosen distance then only $K / \pi r^{2}, M, K_{\text {inhom }}$ and $K_{m m}$ can be used.

To conclude, the literature is not yet stabilized on the criteria that any distance-based method should respect (Combes and Overman, 2004; Bonneu and Thomas-Agnan, 2015). Broadly speaking, methods treating space as continuous are still in their infancy in economics in that they have only been developed over the past decade. As Combes and Overman (2004) stressed, improvements are still needed because no distance-based method is bound to a theoretical model to explain agglomeration. The debate is still open as to the "best" candidate for detecting spatial structures in economics as noticed by Gibbons et al. (2015) even if we have shown that using different measures could bring a more complete analysis of the data. The next step in the analysis is to provide a complete theoretical background for distance-based methods. The statistical literature (Møller and Waagepetersen, 2004) mainly deals with homogeneous point processes, used for topographic measures. Many theoretical results exist, such as $g$ and $K$ expectations for many processes (Diggle, 1983; Illian et al., 2008). For the moment, topographic measures for inhomogeneous space and relative measures still lack statistical support. This could be explained by the long tradition in various scientific domains of employing (and thus developing) functions based on homogeneous point processes. ${ }^{7}$ Since the use of distance-based measures is more recent in economics, theoretical developments are just beginning. More are needed to improve the statistical background of the distance-based measures in a spatial economic framework. First studies are being done in that direction. The growing interest in the $K_{d}$ measure is very promising (Ellison et al., 2010; Alfaro and Chen, 2014) and theoretical developments are now proposed (Barlet et al., 2013). It is also the case of the $M$ function: it has been proved recently that $M$ is the generalization of Ripley's function with inhomogeneous point processes (Marcon et al., 2012).

\subsection{Computation of distance-based methods}

One important point concerns the computation of all these functions and their respective confidence intervals, which can be very time-consuming. Quite often the important computational requirements are noticed by the authors (see Kosfeld et al., 2011, among others). Recent developments have been proposed (Scholl and Brenner, 2015). For example, an easy-to-use package called dbmss for "distance-based measures of spatial structures" (Marcon et al., 2015 ) is now developed, based on the R spatstat package. ${ }^{8}$ This package allows the simple computation of all distance-based measures presented in this article.

Distance-based methods are computer intensive. Complexity, resulting in computation time, is proportional to the squared number of points (to calculate distances between all pairs of points) and the number of simulations of the null hypothesis. Memory requirement is proportional to the squared number of points. The number of points involved in computation depends on the function. Often, the spatial structure of a single type of points (say, a sector of activity) is studied. Topographic and absolute measures only consider the points of this sector; the other points are

\footnotetext{
${ }^{7}$ This is the case for example in forestry where relative measures have not been employed before Marcon et al. (2012).

${ }^{8}$ Available at the following address: http: / / cran.r-project.org/web/packages/dbmss /
} 
either ignored (topographic measures) or used as possible locations in the null hypothesis. Relative measures take into account all points in their benchmark so their computation time is much longer: if the type of interest contains $10 \%$ of the points, computing $K_{d}$ is roughly 100 times faster than computing $m$. Reducing the memory requirement is possible with approximation techniques (Scholl and Brenner, 2015) to summarize the $n(n-1) / 2$ distances values by classes, making it virtually independent of the size of the point pattern. This opens the door to deal with point patterns of several tenths of thousands (computed) points with a current laptop computer. Hundred of thousands points will require days or weeks of computation time that can be parallelized by the dbmss package if the necessary hardware is available. On this subject, Marcon et al. (2015) provide a specific time-requirement example using the dbmss $\mathrm{R}$ package.

To conclude, recent improvements in the computation of distance-based methods are very good news. The intensive and somewhat unfriendly computing was noticed as a limit of the use of distance-based methods in comparison to the Gini or the Ellison and Glaeser indices.

Distance-based methods are also data intensive. The availability of micro-geographic data has increased a lot (Arbia, 2001a) but they may sometimes be blurred for confidentiality or technical reasons. What are the consequences of the uncertainty about the exact location of entities on distance-based measures? Only a small number of studies have tested the potential effects on the results of distance-based methods. On very limited datasets in ecology, Fehmi and Bartolome (2001) show that differences in the $K$ plots can be detected if they use the exact position of the plants or a small grid-based data. Studying the mechanical engineering industry in the metropolitan France, Barlet et al. (2008) do not notice that introducing a geographical noise affect $K_{d}$ plots. More exactly, the use of the exact position of the plants or their position at the municipality level (commune level) visually lead to the same results $\left(K_{d}\right.$ plots are superposed for all distances from $0 \mathrm{~km}$ to $1000 \mathrm{~km}$ ). As far as we know, no theoretical derivation of the uncertainty or systematic simulation study has been yet conducted for distance-based measures. This will certainly be a future way of research and the results will help to grasp the importance of the MAUP issues (as we presented in the introduction of the paper). For the moment, we can advance two points. First, density functions use kernel estimation of the number of neighbors: relatively small errors in the distances could very likely have a limited effect on the estimation and compensate across pairs of points. Second, cumulative functions are insensitive to errors at smaller scales than the distance they consider: if the uncertainty is a few hectometers, the number of neighbors up to a few kilometers is known with no error except for the more distant ones, which are a small proportion.

\subsection{Alternative distances}

All the methods presented above rely on Euclidean distances to define neighborhoods. Alternative distances have been discussed (Yamada and Thill, 2004; Combes and Lafourcade, 2005) to take into account the real-world geography, where streets and roads define the paths between points and bridges are necessary to cross rivers. The network- $K$ function has been defined and studied by Okabe and Yamada (2001): it applies to distances measured along a street network. The mathematical properties of point processes generally rely on Euclidean distances so many known results (such as the expectation of the $K$ function under CSR) are lost when this constraint is released.

Distances may also be defined in a graph as the minimum the number of steps between two nodes. The $M$ function can be adapted easily by defining that a neighborhood is the set of the $n$ nearest neighbors of a reference point rather than the points less than $r$ apart from it. Taking $n=1$ (i.e., considering the nearest neighbor of each point only) and setting all weights to 1, $M$ becomes the colocation quotient of Leslie and Kronenfeld (2011). These possibilities have been little explored in the empirical literature (but see Bentley et al., 2016).

\section{Conclusion}

A decade ago, disproportionality methods such as the Gini or Ellison and Glaeser indices were classical tools for economists. Quite logically, methods were then developed to take advantage of the knowledge of the exact position of objects and solve issues linked to the Modifiable Areal Unit Problem (Openshaw and Taylor, 1979). The first were statistics based on the distance of the nearest neighbor of points, after Clark and Evans (1954). They have been outdated by the distance-based measures of concentration reviewed in this paper because the latter use the information provided by all points less than $r$ apart from each reference point instead of just one. 
When geo-referenced data are available, distance-based measures of concentration are a complete set of tools to test data against null hypotheses of independence (to show aggregation or repulsion) and, for some of them, to quantify the phenomena. We have explained in this article (table 6) which tool to use according to the underlying framework (topographic, absolute or relative). Topographic measures are widely used and updated by ecologists in handbooks (Fortin and Dale, 2005; Illian et al., 2008) which ignore relative measures. Economists mainly use absolute and relative measures to take into account of the overall distribution of economic activities. Several economists (among others Combes et al., 2008) clearly state that applications of distance-based methods should now be given preference by researchers. The problem of the availability of geo-referenced economic data or easy-to-use programs to implement these functions are short-term issues (Overman, 2008), although they have not been solved yet (Marcon et al., 2015). However, relating these descriptive tools to economic theory is the real challenge (Alfaro and Chen, 2014; Kerr and Kominers, 2015), following the way opened by Ellison et al. (2010).

\section{Acknowledgments}

We thank the editor, two anonymous referees and participants at the $61^{\text {st }}$ Congress of the French Economic Association (Paris), Hotelling Seminar (Université de Paris Sud / ENS Cachan) and the $12^{\text {th }}$ International Workshop Spatial Econometrics and Statistics (Orléans, France). The second author gratefully acknowledges financial support from the LET (Université de Lyon, CNRS, ENTPE), IUT de Sceaux and AAP Attractivité 2014 (Université de Paris-Sud). This work has benefited from an "Investissement d'Avenir" grant managed by Agence Nationale de la Recherche (CEBA, ref.ANR-10-LABX-0025).

\section{References}

Alfaro L, Chen MX (2014). "The global agglomeration of multinational firms." Journal of International Economics, 94(2), 263-276.

Arbia G (1989). Spatial Data Configuration in Statistical Analysis of Regional Economic and Related Problems. Kluwer, Dordrecht.

Arbia G (2001a). "Modelling the Geography of Economic Activities on a Continuous Space." Papers in Regional Science, 80(4), 411-424.

Arbia G (2001b). "The Role of Spatial Effects in the Empirical Analysis of Regional Concentration.” Journal of Geographical Systems, 3(3), 271-281.

Arbia G, Copetti M, Diggle P, Fratesi U, Senn L (2009). "Modelling Individual Behaviour of Firms in the Study of Spatial Concentration.”, In U Fratesi, L Senn (eds.), Growth and Innovation of Competitive Regions, pp. 297-327. Advances in Spatial Science, second edition. Springer, Berlin.

Arbia G, Espa G (1996). Statistica economica territoriale. Cedam, Padua.

Arbia G, Espa G, Giuliani D, Mazzitelli A (2012). "Clusters of firms in an inhomogeneous space: The high-tech industries in Milan.” Economic Modelling, 29(1), 3-11.

Arbia G, Espa G, Quah D (2008). "A class of spatial econometric methods in the empirical analysis of clusters of firms in the space." Empirical Economics, 34(1), 81-103.

Baddeley AJ, Møller J, Waagepetersen RP (2000). "Non- and semi-parametric estimation of interaction in inhomogeneous point patterns." Statistica Neerlandica, 54(3), 329-350.

Baddeley AJ, Turner R (2005). "Spatstat: an R package for analyzing spatial point patterns." Journal of Statistical Software, 12(6), 1-42. 
Barff RA (1987). "Industrial Clustering and the Organization of Production: A Point Pattern Analysis of Manufacturing in Cincinnati, Ohio." Annals of the Association of American Geographers, 77(1), 89-103.

Barlet M, Briant A, Crusson L (2008). “Concentration géographique dans l'industrie manufacturière et dans les services en France : une approche par un indicateur en continu." Série des documents de travail de la Direction des Études et Synthèses Économiques G 2008 / 09, Institut National de la Statistique et des Études Économiques (Insee).

Barlet M, Briant A, Crusson L (2013). "Location patterns of service industries in France: A distance-based approach." Regional Science and Urban Economics, 43(2), 338-351.

Behrens K, Bougna T (2015). "An anatomy of the geographical concentration of Canadian manufacturing industries." Regional Science and Urban Economics, 51, 47-69.

Bentley GC, McCutcheon P, Cromley RG, Hanink DM (2016). "Fitzgerald: A Return to the Neighborhood and Its Contemporary Structural and Geographical Contexts." The Professional Geographer, 68, 414-426.

Besag JE (1977). “Comments on Ripley’s paper.” Journal of the Royal Statistical Society, B 39(2), 193-195.

Bickenbach F, Bode E (2008). "Disproportionality Measures of Concentration, Specialization, and Localization." International Regional Science Review, 31(4), 359-388.

Bonneu F (2007). "Exploring and Modeling Fire Department Emergencies with a Spatio-Temporal Marked Point Process." Case Studies in Business, Industry and Government Statistics, 1(2), 139-152.

Bonneu F, Thomas-Agnan C (2015). "Measuring and Testing Spatial Mass Concentration with Micro-geographic Data." Spatial Economic Analysis, 10(3), 289-316.

Briant A, Combes PP, Lafourcade M (2010). "Dots to boxes: Do the Size and Shape of Spatial Units Jeopardize Economic Geography Estimations?” Journal of Urban Economics, 67(3), 287-302.

Brülhart M, Traeger R (2005). "An Account of Geographic Concentration Patterns in Europe.” Regional Science and Urban Economics, 35(6), 597-624.

Ciccone A, Hall RE (1996). "Productivity and the Density of Economic Activity." The American Economic Review, 86(1), 54-70.

Clark PJ, Evans FC (1954). "Distance to nearest neighbor as a measure of spatial relationships in populations." Ecology, 35(4), 445-453.

Combes PP, Lafourcade M (2005). "Transport costs: Measures, determinants, and regional policy implications for France." Journal of Economic Geography, 5(3), 319-349.

Combes PP, Mayer T, Thisse JF (2008). Economic Geography, The Integration of Regions and Nations. Princeton University Press, Princeton.

Combes PP, Overman HG (2004). "The spatial distribution of economic activities in the European Union." In JV Henderson, JF Thisse (eds.), Handbook of Urban and Regional Economics, volume 4, chapter 64, pp. 2845-2909. Elsevier. North Holland, Amsterdam.

Cressie NA (1993). Statistics for spatial data. John Wiley \& Sons, New York.

Cutrini E (2009). "Using entropy measures to disentangle regional from national localization patterns." Regional Science and Urban Economics, 39(2), 243-250. 
Deurloo MC, De Vos S (2008). "Measuring segregation at the micro level: an application of the M measure to multi-ethnic residential neighbourhoods in Amsterdam." Tijdschrift voor economische en sociale geografie, 99(3), $329-347$.

Diggle PJ (1983). Statistical analysis of spatial point patterns. Academic Press, London.

Diggle PJ (1985). “A Kernel Method for Smoothing Point Process Data.” Applied Statistics, 34(2), 138-147.

Diggle PJ, Chetwynd AG (1991). "Second-Order Analysis of Spatial Clustering for Inhomogeneous Populations." Biometrics, 47(3), 1155-1163.

Diggle PJ, Gomez-Rubio V, Brown PE, Chetwynd AG, Gooding S (2007). "Second-order analysis of inhomogeneous spatial point processes using case-control data.” Biometrics, 63(2), 550-557.

Duranton G (2008). "Spatial Economics.", In SN Durlauf, LE Blume (eds.), The New Palgrave Dictionary of Economics. Palgrave Macmillan.

Duranton G, Overman HG (2002). “Testing for Localization Using Micro-Geographic Data.” Discussion Paper 3379, CEPR.

Duranton G, Overman HG (2005). "Testing for Localization Using Micro-Geographic Data." Review of Economic Studies, 72(4), 1077-1106.

Duranton G, Overman HG (2008). "Exploring the Detailed Location Patterns of UK Manufacturing Industries using Microgeographic Data.” Journal of Regional Science, 48(1), 213-243.

Ellison G, Glaeser EL (1997). "Geographic Concentration in U.S. Manufacturing Industries: A Dartboard Approach." Journal of Political Economy, 105(5), 889-927.

Ellison G, Glaeser EL, Kerr WR (2010). "What Causes Industry Agglomeration? Evidence from Coagglomeration Patterns." The American Economic Review, 100(3), 1195-1213.

Fehmi JS, Bartolome JW (2001). "A grid-based method for sampling and analysing spatially ambiguous plants." Journal of Vegetation Science, 12(4), 467-472.

Fernandez-Gonzalez R, Barcellos-Hoff MH, de Solorzano CO (2005). "A Tool for the Quantitative Spatial Analysis of Complex Cellular Systems.” IEEE Transactions on Image Processing, 14(9), 1300-1313.

Feser EJ, Sweeney SH (2000). "A test for the coincident economic and spatial clustering of business enterprises." Journal of Geographical Systems, 2(4), 349-373.

Florence PS (1972). The Logic of British and American Industry: A Realistic Analysis of Economic Structure and Government. 3rd edition. Routledge \& Kegan Paul, London.

Fortin MJ, Dale MRT (2005). Spatial Analysis. A guide for ecologists. Cambridge University Press, Cambridge.

Fratesi U (2008). "Issues in the measurement of localization." Environment and Planning A, 40(3), 733-758.

Gibbons S, Overman HG, Pattachini E (2015). "Spatial methods.” In G Duranton, J Henderson, WC Strange (eds.), Handbook of Urban and Regional Economics, volume 5A, chapter 3, pp. 115-168. Elsevier. North Holland, Amsterdam.

Gini C (1912). Variabilità e mutabilità, volume 3. Università di Cagliari.

Giuliani D, Arbia G, Espa G (2014). "Weighting Ripley’s K-Function to Account for the Firm Dimension in the Analysis of Spatial Concentration.” International Regional Science Review, 37(3), 251-272. 
Goreaud F, Pélissier R (1999). “On explicit formulas of edge-effect correction for Ripley's K-function.” Journal of Vegetation Science, 10(3), 433-438.

Goreaud F, Pélissier R (2003). "Avoiding misinterpretation of biotic interactions with the intertype $K_{12}$ function: population independence vs random labelling hypotheses.” Journal of Vegetation Science, 14(5), 681-692.

Guillain R, Le Gallo J (2010). "Agglomeration and Dispersion of Economic Activities in and around Paris: An Exploratory Spatial Data Analysis.” Environment and Planning B, 37(6), 961-981.

Guimarães P, Figueiredo O, Woodward D (2011). “Accounting for neighboring effects in measures of spatial concentration.” Journal of Regional Science, 51(4), 678-693.

Heinrich L (1991). "Goodness-of-fit tests for the second moment function of a stationary multidimensional poisson process.” Statistics: A Journal of Theoretical and Applied Statistics, 22(2), 245 - 268.

Illian J, Penttinen A, Stoyan H, Stoyan D (2008). Statistical Analysis and Modelling of Spatial Point Patterns. Statistics in Practice. Wiley-Interscience, Chichester.

Jensen P, Michel J (2011). "Measuring spatial dispersion: exact results on the variance of random spatial distributions." The Annals of Regional Science, 47(1), 81-110.

Kerr WR, Kominers SD (2015). “Agglomerative Forces and Cluster Shapes.” The Review of Economics and Statistics, 97(4), 877-899.

Kiskowski MA, Hancock JF, Kenworthy AK (2009). “On the Use of Ripley's K-Function and Its Derivatives to Analyze Domain Size.” Biophysical Journal, 97(4), 1095-1103.

Klier T, McMillen DP (2008). "Evolving agglomeration in the U.S. auto supplier industry." Journal of Regional Science, 48(1), 245-267.

Koh HJ, Riedel N (2014). "Assessing the Localization Pattern of German Manufacturing and Service Industries: A Distance-based Approach.” Regional Studies, 48(5), 823-843.

Kosfeld R, Eckey HF, Lauridsen J (2011). "Spatial point pattern analysis and industry concentration.” The Annals of Regional Science, 47(2), 311-328.

Lagache T, Lang G, Sauvonnet N, Olivo-Marin JC (2013). "Analysis of the Spatial Organization of Molecules with Robust Statistics.” Plos One, 8(12), e80914.

Lang G, Marcon E (2013). "Testing randomness of spatial point patterns with the Ripley statistic." ESAIM: Probability and Statistics, 17, 767-788.

Lang G, Marcon E, Puech F (2015). "Distance-Based Measures of Spatial Concentration: Introducing a Relative Density Function.” HAL, hal-01082178(version 2).

Law R, Illian J, Burslem D, Gratzer G, Gunatilleke CVS, Gunatilleke I (2009). "Ecological information from spatial patterns of plants: insights from point process theory." Journal of Ecology, 97(4), 616-628.

Leslie TF, Kronenfeld BJ (2011). "The Colocation Quotient: A New Measure of Spatial Association Between Categorical Subsets of Points." Geographical Analysis, 43(3), 306-326.

Loop MS, McClure LA (2015). "Testing for clustering at many ranges inflates family-wise error rate (FWE)." International Journal of Health Geographics, 14(4).

Loosmore NB, Ford ED (2006). "Statistical inference using the G or K point pattern spatial statistics." Ecology, 87(8), 1925-1931. 
Lotwick HW, Silverman BW (1982). "Methods for Analysing Spatial Processes of Several Types of Points." Journal of the Royal Statistical Society, 44(3), 406-413.

Marcon E, Puech F (2003). "Evaluating the Geographic Concentration of Industries Using Distance-Based Methods." Journal of Economic Geography, 3(4), 409-428.

Marcon E, Puech F (2010). "Measures of the Geographic Concentration of Industries: Improving Distance-Based Methods." Journal of Economic Geography, 10(5), 745-762.

Marcon E, Puech F (2015). "Mesures de la concentration spatiale en espace continu : théorie et applications." Économie et Statistique, 474, 105-131.

Marcon E, Puech F, Traissac S (2012). "Characterizing the relative spatial structure of point patterns.” International Journal of Ecology, 2012(Article ID 619281), 11.

Marcon E, Traissac S, Lang G (2013). "A Statistical Test for Ripley's Function Rejection of Poisson Null Hypothesis." ISRN Ecology, 2013(Article ID 753475), 9.

Marcon E, Traissac S, Puech F, Lang G (2015). "Tools to Characterize Point Patterns: dbmss for R." Journal of Statistical Software, 67(3), 1-15.

Møller J, Waagepetersen RP (2004). Statistical Inference and Simulation for Spatial Point Processes, volume 100 of Monographs on Statistics and Applies Probabilities. Chapman and Hall.

Myllymäki M, Mrkvicka T, Grabarnik P, Seijo H, Hahn U (2015). “Global envelope tests for spatial processes.” arXiv, 1307.0239(version 4).

Nakajima K, Saito YU, Uesugi I (2012). "Measuring economic localization: Evidence from Japanese firm-level data." Journal of the Japanese and International Economies, 26(2), 201-220.

Ngo Bieng MA, Ginisty C, Goreaud F (2011). "Point process models for mixed sessile forest stands." Annals of Forest Science, 68(2), 267-274.

Nissi E, Sarra A, Palermi S, Luca G (2013). "The Application of M-Function Analysis to the Geographical Distribution of Earthquake Sequence.”, In A Giusti, G Ritter, M Vichi (eds.), Classification and Data Mining, chapter 32, pp. 271-278. Studies in Classification, Data Analysis, and Knowledge Organization. Springer Berlin Heidelberg.

Ó hUallacháin B, Leslie TF (2007). "Producer Services in the Urban Core and Suburbs of Phoenix, Arizona." Urban Studies, 44(8), 1581-1601.

Ohser J (1983). “On estimators for the reduced second moment measure of point processes." Series Statistics, 14(1), 63-71.

Okabe A, Yamada I (2001). "The $K$-function method on a network and its computational implementation." Geographical Analysis, 33(3), 271-290.

Openshaw S, Taylor PJ (1979). "A million or so correlation coefficients: three experiments on the modifiable areal unit problem.”, In N Wrigley (ed.), Statistical Applications in the Spatial Sciences, pp. 127-144. Pion, London.

Overman HG (2008). "GIS data in economics." The New Palgrave Dictionary of Economics, (Second Edition).

Penttinen A (2006). "Statistics for Marked Point Patterns.", In The Yearbook of the Finnish Statistical Society, pp. 70-91. The Finnish Statistical Society, Helsinki. 
Penttinen A, Stoyan D, Henttonen HM (1992). "Marked Point Processes in Forest Statistics." Forest Science, 38(4), 806-824.

Pélissier R (1998). "Tree spatial patterns in three contrasting plots of a southern Indian tropical moist evergreen forest." Journal of Tropical Ecology, 14(1), 1-16.

R Core Team (2016). R: A Language and Environment for Statistical Computing. R Foundation for Statistical Computing. ISBN 3-900051-07-0.

Ripley BD (1976). "The Second-Order Analysis of Stationary Point Processes.” Journal of Applied Probability, 13(2), 255-266.

Ripley BD (1977). “Modelling Spatial Patterns.” Journal of the Royal Statistical Society, B 39(2), 172-212.

Rowlingson B, Diggle (1993). "SPLANCS: Spatial Point Pattern Analysis Code in S-Plus." Computers \& Geosciences, 19(5), 627-655.

Scholl T, Brenner T (2015). "Optimizing distance-based methods for large data sets." Journal of Geographical Systems, 17(4), 333-351.

Silverman BW (1986). Density estimation for statistics and data analysis. Chapman and Hall, London.

Stoyan D, Ohser J (1984). “Cross-correlation measures for weighted random measures.” Teoriya Veroyatnostei i ee Primeneniya, 29(2), 338-347.

Stoyan D, Ohser J (1985). "Cross-Correlation Measures of Weighted Random Measures and Their Estimation." Theory of Probability and its Applications, 29(2), 345-355.

Stoyan D, Stoyan H (2000). "Improving ratio estimators of second order point process characteristics." Scandinavian Journal of Statistics, 27(4), 641-656.

Sweeney SH, Feser EJ (1998). "Plant Size and Clustering of Manufacturing Activity." Geographical Analysis, 30(1), $45-64$.

Waller L (2010). "Point Process Models and Methods in Spatial Epidemiology.", In A Gelfand, P Diggle, P Guttorp, M Fuentes (eds.), Handbook in Spatial Statistics, chapter 22, pp. 403-423. CRC Handbooks of Modern Statistical Methods Series. Chapman \& Hall.

Wiegand T, Moloney KA (2004). "Rings, circles, and null-models for point pattern analysis in ecology." Oikos, 104(2), 209-229.

Wiegand T, Moloney KA, Naves J, Knauer F (1999). "Finding the Missing Link between Landscape Structure and Population Dynamics: A Spatially Explicit Perspective.” The American Naturalist, 154(6), 605-627.

Yamada I, Thill JC (2004). "Comparison of planar and network $K$-functions in traffic accident analysis." Journal of Transport Geography, 12(2), 149-158. 